\title{
La particularité du jugement d'ouvrir la procédure de faillite économique dans le règlement de faillite saoudien (Étude analytique)
}

\author{
Adnan salih Alomar \\ Professeur du droit commercial \\ Facult é de droit, Université de Yarmouk, Jordan \\ adnan.alomar@yu.edu.jo
}

\author{
Ali Alhamdan \\ Ancien professeur du droit international \\ à l'Université de Damas, Syria \\ alialhamdan92@gmail.com
}

Date de réception: $25 / 2 / 2020$

Date d'acceptation: 30/03/2020

DOI: https://doi.org/DOI:10.31559/LCJS2020.1.1.4

Résumé: La faillite est sans aucun doute l'une des garanties fondamentales pour protéger le crédit et la confiance dans les opérations commerciales, et l'idée du concept de faillite était auparavant limitée à une activité commerciale et non à d'autres activités économiques. Cependant, le législateur saoudien dans le nouveau système de faillite saoudien pour l'année $1439 \mathrm{AH}$, a adopté l'idée de soumettre tous les projets économiques visant à réaliser des profits matériels à la portée de la faillite, ce qui nous a incité à sélectionner le terme de "faillite économique". Donc, la législation est désormais en conforme avec la loi type de la CNUDCI sur l'insolvabilité de 1997, ainsi qu'avec de nombreuses lois étrangères et arabes.

Par contre, le législateur saoudien s'est écarté du principe général de la faillite : il n'exige plus de décision judiciaire pour mettre en faillite le débiteur qui a cessé de payer ses dettes, mais il a proposé un certain nombre de procédures, allant du règlement préventif à la liquidation, et stipulant que chacune de ces procédures pouvait être soumise à des conditions objectives et formelles. Cela nous a amenés à examiner les conditions générales de ces procédures dans le système de faillite saoudien et à déterminer dans quelle mesure elles sont appropriées pour demander l'une de ces procédures. L'étude a abouti à un certain nombre de recommandations, dont certaines sous la forme de propositions de modification du texte des articles pertinents, notamment la nécessité de trouver un équilibre entre les intérêts des débiteurs et ceux des créanciers, ainsi de donner au parquet le droit de demander l'ouverture de la procédure de liquidation lorsque les conditions pour son ouverture sont remplies.

Mots-clés : défaillance financière ; règlement préventif; liquidation ; réorganisation financière ; actifs investis.

\section{Introduction}

La faillite découle de la prononciation d'un jugement de déclaration de faillite rendu par le tribunal compétent pour statuer dans les actions faillite, il s'agit selon le nouveau règlement (Code) judiciaire du tribunal commercial de la circonscription où réside le défendeur. En se référant à l'article 25 de ce nouveau règlement judiciaire, le tribunal de commerce disposes de la compétence générale pour juger toutes les affaires commerciales, y compris les actions relatives à la faillite et à la mise des faillis en quarantaine.

La compétence ratione loci du tribunal où est déclarée la faillite, à savoir le tribunal de la circonscription de résidence du débiteur, correspond au lieu où siège l'administration de l'entreprise de l'individu débiteur, tandis qu'elle correspond au centre des affaires économiques des institutions, des entreprises et des établissements économiques. En effet, le législateur confie au tribunal de faillite la compétence de statuer dans les différents qui lui sont relatifs ou qui en découlent, qu'ils soient civils ou commerciaux, relatifs à un bien ou un immobilier, et ceci en application de la règle qui dit que le juge principal est lui-même l'officier judiciaire sous-divisionnaire.

Auparavant le législateur saoudien exigeait l'obligation de la prononciation d'un jugement judiciaire déclarant la faillite ; hors celui-ci a déraillé de cette règle lorsqu'il a changé de position c'est qu'en cas de faillite du débiteur, le législateur n'a pas exigé de décision judiciaire déclarant sa faillite ; mais il s'est limité à ce que ce débiteur soit incapable de payer ses dettes à leurs termes pour lancer les diverses procédures de faillite, commençons par la procédure de règlement préventif, de la liquidation des fonds du débiteur failli tout en passant par le redressement financier. Ces procédures sont menées sous la supervision judiciaire afin de garantir leur bon fonctionnement, la gestion et l'administration de la saisi ainsi que d'assurer la répartition du net des avoirs sur les créanciers. 


\section{L'intérêt de l'étude}

L'intérêt de la présente étude réside dans la volonté de préciser la nature de la décision de lancement des procédures de la faillite et ses caractéristiques, de préciser également la différence entre celle-ci et le jugement de déclaration de la faillite, de même qu'entre les jugements rendus des diverses procédures de faillites qui ont été traitées par le législateur saoudien au niveau du nouveau règlement de faillite. Allant du règlement préventif à la liquidation tout en passant par le redressement financier. En effet, le législateur saoudien exige la nécessité de commencer par la demande de l'une de ces différentes procédures, la nécessité de prononciation d'un jugement du tribunal compétent, sachant bien que le jugement de chacune d'elles varient selon le demandeur du jugement, les conditions que la demande et les effets qui en découlent doivent remplir.

\section{Les objectifs de l'étude}

Cette étude cherche à mettre en lumière la nature du jugement de lancement des procédures de faillite déterminée par le législateur saoudien dans le nouveau règlement de faillite, ainsi qu'elle vise la précision du niveau de sa conformité audit règlement qui cible l'animation de la solvabilité, le renforcement de la confiance au niveau des transactions commerciales à travers une gamme de procédures et de règles susceptibles d'aider le débiteur et de sauver son projet de la faillite, ainsi que de protéger les créanciers en protégeant leurs droits, et ceci en leur permettant de saisir le reste des fonds du débiteur en les mettant entre les mains de la justice ; l'objectif étant donc la protection des intérêts des créanciers, la garantie de l'égalité entre eux ainsi que l'organisation de la répartition des fonds du débiteur qui leur sont distribués pour une répartition équitable ne favorisant aucun créancier à un autre tant que sa dette n'est pas liée à un motif juridique exigeant ce privilège.

\section{La problématique}

Comme le législateur saoudien n'a pas exigé dans le nouveau règlement de faillite un verdict de faillite tel que c'était le cas auparavant, où elles commençaient par un unique jugement de faillite décidant de l'insolvabilité du débiteur incapable de payer ses dettes. Toutefois, dans le nouveau règlement de faillite, le législateur saoudien exige différents jugements qui varient en fonction de la procédure voulue. Dans ce contexte, la problématique de la présente étude est la précision de la nature et de la spécificité de ces jugements rendus afin de lancer l'une des procédures de faillite, à commencer par la procédure du règlement préventif, puis le jugement de redressement financier et enfin le jugement portant sur la liquidation mettant fin aux activités du débiteur et la répartition de ses fonds aux créanciers équitablement.

\section{Les questions de l'étude:}

En guise de déterminer la problématique susmentionnée, il est indispensable de la formuler en des questions auxquels nous devons apporter des éléments de réponse, il s'agit de:

- Quel est le degré d'importance de l'exigence d'un jugement en cas de faillite?

- Quelle est la différence entre le jugement unique de déclaration de la faillite et les jugements relatifs au lancement des diverses procédures de faillite?

- Quel est le mécanisme d'exécution des jugements de lancement des procédures de faillite et les moyens d'en faire appel.

\section{La méthodologie de l'étude:}

Comme cette étude est limitée à la précision de la spécificité des jugements rendus pour lancer les procédures de faillite sous le nouveau règlement de la faillite de 1439 de l'hégire, nous nous basons dans la présente étude sur la méthodologie analytique descriptive des articles de ce règlement qui sont en rapport avec la question objet de notre étude, et ceci dans la perspective de préciser son degré d'efficacité et de cohérence avec le cadre général de l'organisation juridique de la faillite.

\section{Le plan de l'étude:}

L'étude est scindée en deux chapitres, à savoir:

Chapitre1: La demande d'engagement d'une procédure de faillite économique.

Section1: Le tribunal compétent pour demander le lancement de la procédure.

Section2: Le droit à la demande de lancement de la procédure. 
Chapitre2: La nature juridique du jugement rendu pour lancer une procédure de faillite.

Section1: Les caractéristiques du jugement de la procédure de faillite.

Section2: Le contenu du jugement de la procédure de faillite.

\section{Préambule:}

Pour lancer l'une des procédures de faillite, le législateur saoudien exige un jugement rendu par le tribunal compétent de l'ouverture des procédures de faillite, ce qui correspond au contenu de l'article (41) du nouveau règlement de faillite qui stipule que : «Le tribunal décide de sa propre initiative ou sur demande d'une partie intéressée d'ouvrir la procédure adéquate de faillite si les conditions suivantes sont remplies... », d'ailleurs, c'est ce qui a été confirmé par les articles $(15,47,90$ et 99) du règlement précité. Ce jugement suscite alors de nombreuses questions juridiques; sur le plan du procès de demande de lancement d'une procédure de faillite, de la connaissance de la nature du jugement d'ouverture de l'une de ses procédures, de la détermination du tribunal compétent pour le rendre, ainsi que sur le plan de la manière de demander cette ouverture, voire le contenu de ce jugement et la façon de le publié, le déclaré et d'en faire appel. Ces thématiques sont toutes discutées dans le présent travail à travers deux chapitres, à savoir:

\section{Chapitre1: La demande d'engagement d'une procédure de faillite économique}

Pour demander le lancement d'une procédure de faillite, le législateur exige que la partie intéressée engage un procès auprès du tribunal juridiquement compétent, selon les procédures juridiques définies, qui se distinguent des autres règles procédurales générales des procès. A travers notre étude, nous estimons expliquer le procès de demande de lancement d'une procédure de faillite, et ceci en étudiant les questions suivantes:

\section{Section1: Le tribunal compétent pour demander le lancement de la procédure}

Pour lancer l'une des procédures de faillite, une décision judiciaire du tribunal compétent d'examiner les procès de faillite est obligatoire, il s'agit selon le nouveau règlement judiciaire du tribunal de commerce de la circonscription du lieu de résidence du défendeur.

\section{- La Ratione_materiæ (qualitative)}

D'après le contenu de l'article 25 du nouveau règlement judiciaire, le tribunal de commerce a la compétence générale de statuer de toutes les affaires commerciales, y compris celles de la faillite et de la mise en quarantaine des faillis (Karman, 1429 de l'hégire, p :317). En effet, ceci a été consacré par le législateur saoudien au niveau du troisième paragraphe du premier article du nouveau règlement de faillite où il définit le tribunal compétent étant «le tribunal de commerce».

Evidemment, la Ratione materiæ de statuer des affaires émanant des cas de faillite revient au tribunal de commerce ne susciterait aucune problématique autour de son mérite d'examiner ce type de procès, compte tenu du fait que le cas de faillite se limitait aux commerçants seulement, ces derniers qui sont alors soumis aux tribunaux de commerce spécialisés à l'origine dans l'examen de toute affaire relative à leurs transactions, y compris les actions de faillite.

Hors, suite à la nouvelle position qu'adopte le législateur en termes du nouveau règlement de faillite qui diffère de son précédent, le champs d'application de ce règlement ne se limite plus aux commerçants seuls mais de plus de ceux-ci il comprend toutes les personnes, entités et institutions à but lucratif ; ce qui signifie la possibilité de faillite de personnes n'ayant pas la qualité commerçante, qui continuent d'exercer des métiers et activités non commerciales; comme les personnes qui exercent des métiers professionnels, industriels ou civils, etc. Par conséquent, ces derniers sont soumis au tribunal de commerce en ce qui concerne les procès de faillite.

Incontestablement, ceci constitue une dérogation à la règle générale prévoyant que les non-commerçants ne peuvent être soumis aux tribunaux de commerce. Ainsi, le législateur saoudien a élargi le champ du droit commercial afin d'inclure toutes les activités économiques, industrielles et professionnelles considérées comme affluents du service commercial dans l'Etat.

Cependant, la ratione materiæ/ la compétence qualitative du tribunal de commerce une partie de la réglementation général, car il est interdit de se mettre d'accord en guise de l'enfreindre ou de l'amender, comme il est possible de le maintenir à une étape quelconque du procès, de même, le tribunal peut le susciter de sa propre initiative (Taha, 1988, p :348) ; Al-Baroudi, 1968, p :284), même si les parties au conflit ne le demande pas. Ceci est expliqué par sa référence à la logique disant que le tribunal est plus capable que quiconque de connaitre la situation financière du débiteur à faillir et liquider, alors une fois la faillite a pris fin, la compétence du tribunal commercial disparait tout en retournant au tribunal compétent selon les règles générales.

En raison de l'intérêt du législateur saoudien porté à l'organisation des dispositions de la faillite en élargissant son champ pour qu'elles incluent toutes les personnes et entités commerciales et toute autre entité à but lucrative, par conséquent la soumission d'autres personnes que les commerçants à ces dispositions-là. En effet, nous 
souhaitions que le législateur saoudien ait stipule la nécessité de créer un tribunal spécifique à la faillite, comme ont fait beaucoup de lois de faillites à travers le monde, pour statuer des questions relatives à la faillite, parmi ses magistrats ceux ayant la maîtrise suffisante de l'ensemble des dispositions juridiques relatives aux commerçants, ainsi qu'à d'autres personnes qui exercent des métiers professionnels, industriels ou de nature économique, qui peuvent différé dans leur fond des conflits de nature commerciale pure et dure (George, 1995, p1491-1501)

\section{- La ratione loci (la compétence territoriale)}

La ratione loci ou la compétence territoriale, dans la mesure où les dispositions générales exigent d'intenter une action auprès du tribunal territorialement compétent, ce territoire où se situe le lieu de résidence du défendeur; que celui-ci soit une personne physique (un individu) ou une personne morale (une entreprise ou un organisme public). En réalité, le législateur saoudien n'a pas apporté de nouveauté en ce qui concerne la détermination du tribunal compétent de statuer des procédures de faillite, car il a gardé la compétence du tribunal de commerce dont la circonscription inclut le lieu de résidence du débiteur et ceci selon les règles générales.

En conséquence, la demande d'ouverture de la procédure doit être adressée au tribunal du lieu de résidence du débiteur s'il s'agit d'une personne physique, ou au tribunal du lieu où se situe administration s'il s'agit d'une personne morale. De notre part, nous pensons que le mieux était que le législateur stipule expressément la présentation de la demande d'ouverture de l'une des procédures de faillite devant le tribunal de la circonscription où exerce le débiteur ses travaux et ses activités à but lucratif et non pas du lieu de sa résidence permanente.

\section{Section2: Le droit à la demande de lancement des procédures de faillite}

Soucieux d'aider du débiteur pour éviter son insolvabilité et l'aider à se remettre, le législateur saoudien fait naître l'espoir des projets en difficulté en permettant au débiteur en difficulté la possibilité de demander au tribunal l'ouverture de la procédure de règlement préventif comme premier pas dans les procédures de faillite, comme il lui a permis ainsi qu'aux créanciers et aux parties responsables de demander le lancement de la procédure de le redressement financier et de la liquidation; pourtant dans ce cas il n'est pas toujours possible de demander la procédure de liquidation du débiteur sauf preuve à travers les informations présentées au tribunal qu'il est impossible de maintenir l'activité de ce débiteur en présence de ces difficultés et que ses avoirs suffisent pour couvrir les dépenses de la procédure de liquidation.

De surcroit, le droit de demander les procédures ne se limite pas uniquement au débiteur, créanciers et aux parties compétentes; mais le législateur saoudien permet expressément au tribunal compétent de décider l'ouverture d'une procédure de faillite de sa propre initiative ou sur demande d'une partie intéressée dès la difficulté du débiteur, ceci est applicable à l'ensemble des procédures de faillite ; telle que le règlement préventif de faillite et le redressement financier, ainsi que la liquidation normale et administrative.

Il ressort clairement de ce qui précède que le droit de demander l'ouverture des procédures de faillite varie d'une procédure à l'autre ; dans certaines il se limite au débiteur en difficulté ou failli, tandis que dans d'autres il concerne aussi bien le débiteur, les créanciers que la partie compétente, comme il peut être un droit du tribunal ou d'une partie intéressée. En contrepartie, ce règlement de faillite n'a pas donné au parquet le droit de demander l'ouverture de certaines procédures de faillite, pour notre part, nous pensons que la procurature générale a ce droit comme nous allons expliquer ultérieurement.

Dans ce qui suit, nous traitons de manière plus détaillée les parties ayant le droit de demander les procédures de faillite.

\section{- La demande d'une procédure de faillite par le débiteur}

Il est indéniable que les procès de faillite sont directement liés au débiteur, à cet effet on trouve que plusieurs articles ont explicitement prévu son droit à demander l'ouverture d'une procédure de faillite quelconque. En conséquence, l'article (13) du nouveau règlement de faillite autorise au débiteur de demander au tribunal compétent l'ouverture de la procédure de règlement préventif une fois que les conditions voulues sont réunies pour ce faire. Certes, faire une telle demande de la part du débiteur témoigne de sa bonne foi, car il connait ses propres conditions et sa situation financière, et donc son incapacité à remplir ses engagements, ce qui signifie qu'il ne vise que la protection des droits de ses créanciers ainsi que le maintien du principe de bonne foi en termes des transactions commerciales (Al-Aekali, 2008, p :61).

De même, l'article (42) dudit règlement a autorisé le débiteur de demander au tribunal le redressement financier selon les conditions fixées par l'article précédent. Le débiteur a aussi le droit de demander l'ouverture de la procédure de liquidation dès qu'il soit en difficulté ou failli, de même que la liquidation administrative si et dans la mesure où ses avoirs suffisent pour couvrir les frais de la procédure de la liquidation normale.

\section{- La demande d'une procédure de faillite par les créanciers}

La demande de lancement de l'une des procédures de faillite par le créancier est la voie normale pour commencer les procédures de faillite visant à garantir les ses droits auprès du débiteur. Le droit du créancier à cette demande est prévu par de nombreux articles du règlement de faillite. A ce titre, l'article (42) stipule le droit du créancier à demander au tribunal la procédure de redressement financier selon les conditions prévues par 
l'article précité, de même il lui est reconnu le droit de présenter une demande d'ouverture de la procédure de liquidation dès la difficulté ou la faillite du débiteur.

Pour demander ces procédures, il n'est exigé au débiteur qu'elles soient demandées par tous les créanciers; il suffit qu'un seul parmi eux les demande, à ce stade le législateur saoudien a bien fait en ajoutant une nouvelle condition à la possibilité de demande de la procédure de liquidation par le créancier, et qui consiste au fait que la valeur de ou des crédit(s) impayé par le débiteur dépasse le minimum arrêté par le comité de faillite pour pouvoir demander ces procédures. Ceci vise à s'assurer de l'incapacité du débiteur failli à regagner sa situation financière de nouveau; ce qui implique sa liquidation dans un tel cas (Boughanmi, 2018, p:233).

Toutefois, on adresse la critique concernant l'exigence du législateur la nécessité d'augmentation du montant du/des crédit(s) de façon à dépasser un seuil donné afin de pouvoir demander la liquidation, cette critique gravite alors autour de l'exigence de prouver le crédit par le créancier par le biais d'un document exécutif, ou encore l'obligation que le crédit soit écrit, que le créancier prouve qu'il a demandé au débiteur de le payer 28 jours avant la date d'inscription de la demande lors qu'il ne l'a pas payé ou bien qu'il sont en conflit à ce propos. Pour s'assurer de la validité du crédit, il n'a pas fallu se limiter à des moyens de preuve bien déterminés; ni de stipuler la possibilité de s'assurer des crédits selon d'autres moyens; notamment le témoignage, le serment, les registres de commerce, présomptions légales, et bien d'autres moyens tenus en compte pour prouver les crédits et les droits, particulièrement en ce qui concerne les crédits commerciaux prouvables par tous les moyens.

Certes, le créancier a le droit de renoncer à sa demande ou de l'abandonner, ou de se contenter d'adresser au débiteur d'honorer sa dette, si le créancier s'attache à sa demande le tribunal devra vérifier que les conditions exigées pour lancer la procédure en question sont remplies; En effet, une fois il s'en assure, le tribunal doit accepter l'ouverture de la procédure de faillite demandée par le créancier selon les règles et les dispositions relatifs à cette procédure.

Nous espérions, que le législateur saoudien ait exigé au demandeur de la procédure, s'il est autre que le débiteur, de payer un montant d'assurance au tribunal qu'il récupère quand sa demande de procédure de faillite est acceptée afin de garantir le sérieux de la demande ainsi que pour éviter de freiner l'activité économique à cause de demandes vindicatives fondées sur des motifs qui ne méritent pas d'être considérés comme de sérieux motifs pour demander l'ouverture d'une procédure de faillite.

\section{- L'ouverture de la procédure de faillite à l'initiative de tribunal}

Les législations de beaucoup de pays autorisent aux tribunaux compétents de statuer des affaires de faillite, de décider la déclaration de la faillite à son initiative sans permission du débiteur ni la demande du créancier, ce qui constitue une dérogation aux règles générales qui interdisent aux tribunaux de se prononcer selon leur autorité ou sans demande qui leur est a été adressée, raison pour laquelle certaines législations ne la tiennent pas en considération ; telle que la législation anglaise et allemande (Taha, 1988, p :390).

En effet, il est hors doute que le motif de cette dérogation est, d'une part, l'attachement des jugements de faillite au règlement général donnant au tribunal le droit de trancher dans les questions relatives au règlement générale à sa propre initiative, même si on ne lui adresse aucune demande. D'autre part, on trouve la protection des intérêts des créanciers, particulièrement les absents ou ceux qui ont été contraints de ne pas faire une demande au tribunal ; à titre d'exemple, si l'un des créanciers demande la déclaration de la faillite puis il renonce à sa demande par collusion avec le débiteur, ou parce qu'il a reçu sa dette, dans ce cas le tribunal a le droit de lancer la procédure de faillite du débiteur, aussitôt que ses conditions sont réunies, l'objectif c'est donc la protection des droits des créanciers absents ou ceux qui n'ont pas adressée une demande au tribunal. En dépit de l'importance d'un tel motif sauf qu'il est difficile pour le tribunal de savoir la situation financière du débiteur en cas d'arrêt de paiement (HUOIN, 1965, p :310).

Auparavant, le législateur saoudien n'a pas prévu explicitement le droit du tribunal à la demande de la faillite du commerçant débiteur à sa propre initiative, excepte dans le cas de nullité du règlement préventif découlant de manœuvres frauduleuses de ce débiteur ; Néanmoins, dans le cadre du nouveau règlement de faillite le législateur a ajusté sa position en autorisant au tribunal de décider l'ouverture de la procédure adéquate de faillite à sa propre initiative ou sur demande d'un intéressé si les conditions suivantes se réunissent... », ce qui est également confirmé par plusieurs autres textes du même règlement.

L'analyse des textes d'articles ayant autorisé au tribunal l'ouverture de la procédure convenable de faillite permet de constater que ces textes ont eu lieu soit suite à l'échec du règlement préventif ou bien du redressement financier à cause des motifs que le législateur a déterminé. En conséquence, à travers l'affaire soumise au tribunal, ce dernier a une idée plus claire qui lui permet de décider l'ouverture la procédure convenable de faillite; notamment, l'ouverture de la procédure de redressement financier ou de liquidation dès que les conditions nécessaires pour ce faire soient remplies. Or, la question qui se pose c'est: est-ce que le tribunal a droit à demander l'ouverture de la procédure du règlement préventif à sa propre initiative ou non? C'est qu'au niveau du nouveau règlement de faillite, nous n'avons trouvé aucun texte dans ce sens; parce que ce droit est reconnu principalement pour l'intérêt du débiteur, car dans le cas où celui-ci ne demande pas le lancement de la procédure parce qu'il est en difficulté ou est failli, ceci risque d'être un réel danger qui menace sa situation et son entité 
économique, qui mène à la liquidation et à la fin de son projet. A ce titre, nous constatons que le législateur saoudien dans l'article (13) précité a limité le droit de demander la procédure de règlement préventif au débiteur seulement pour les motifs susmentionnés.

\section{- L'ouverture de la procédure de faillite sur demande du parquet}

$\mathrm{Au}$ niveau du nouveau règlement de faillite, le législateur saoudien n’a pas prévu expressément le droit du parquet à l'ouverture de l'une des procédures de faillite du débiteur, malgré qu'il s'agisse d'un droit évidemment établi, étant donné que la procurature générale est détentrice de la compétence générale en termes de saisine pour tous les crimes, ce qui a été confirmé par l'article (208) du règlement de faillite qui prévoit que : « le parquet est chargé d'enquête et de poursuite des infractions établies en vertu du règlement, tandis que le tribunal s'occupe des sanctions qui y sont énoncées ». Si le procureur sache que le débiteur avait commis l'un des actes constitutifs d'un délit de faillite; notamment, dissimuler partiellement ou totalement des fonds, le détournement de deniers, l'endommagement de ses livres après avoir cessé de payer, dans ce cas s'il s'avère lors de l'enquête que ce débiteur est en cas d'arrêt de paiement, alors le procureur général a droit de demander l'ouverture de la procédure appropriée de faillite afin de protéger les créanciers.

Nous espérons que le législateur saoudien stipule le droit du parquet à demander la décision d'ouverture de la procédure de faillite appropriée, spécialement celle de la liquidation de l'activité du débiteur failli ou en difficulté, en raison de l'intérêt des créanciers et des services économiques de l’Etat, en général.

\section{Chapitre 2: La nature juridique du jugement rendu pour lancer une procédure de faillite}

Le sixième article du règlement saoudien de faillite stipule que: "Le tribunal rend des jugements et des décisions nécessaires à l'application des procédures prévues par la loi, supervise leur exécution, régit les conflits qui peuvent en découler, ainsi qu'il inflige les sanctions prévues dans le règlement ». D'après le texte de cet article, le tribunal de commerce du lieu de résidence du commerçant débiteur et où ce dernier exerce son commerce, est le compétent pour ouvrir une procédure quelconque de faillite, de la déclarer voire déclarer toutes les dispositions et décision y afférents.

Ceci est consacré par les articles $(41,90,99,140,148,158,156$ et 163) du règlement de faillite, car ces articles soulignent la nécessité de rendement d'un jugement du tribunal compétent décidant l'ouverture d'une procédure de faillite à sa propre initiative ou sur demande d'in intéressé, dès que les conditions nécessaires pour cette procédure soient réunies.

Alors, si le lancement d'une procédure de faillite est tributaire d'une décision judiciaire, cette dernière a certaines caractéristiques qui la distingue des autres décisions normales, en raison des effets importants qu'ils en découlent, ainsi que des nouveaux statuts juridiques qu'elle crée qui n'existaient auparavant. A cet égard, nous tentons à travers cette étude de présenter ce jugement en analysons ses caractéristiques, sa mise en œuvre ainsi que les voies d'en recourir, comme suit:

\section{Section1: Les caractéristiques du jugement de la procédure de faillite}

La nature de la décision d'ouverture d'une procédure quelconque de faillite est déterminée par les caractéristiques fixées les textes réglementaires, il s'agit de:

\section{- L'erga Omnes de la décision d'ouvrir l'une des procédures de faillite}

L'origine dans les jugements judiciaires ordinaires c'est qu'ils reposent sur le principe de la relativité de leur erga Omnes; c'est-à-dire que leurs effets sont généralement limités aux parties au litige, au même sujet ainsi qu'aux mêmes faits (Ahmad, 2001, p: 18-25). En fait, le législateur saoudien a déraillé à cette règle; dans la mesure où son erga Omnes, soit en termes des personnes auxquels il s'applique ou même en termes du sujet qu'il traite.

Cette opposabilité est absolue dans le sens où les effets d'ouverture de la procédure ne se limitent pas uniquement aux parties au conflit; mais plutôt à l'égard de tous, sans distinction entre partie au conflit et autre personne. En effet, le débiteur est considéré soit failli, en difficulté ou bien vulnérable à tomber en difficulté par tous suite au rendement d'un jugement d'ouverture d'une procédure de faillite, ce qui peut varier d'une procédure à une autre. Quant à l'opposabilité du sujet de la procédure, elle est absolue au niveau de la procédure du redressement financier et de la liquidation, car les effets de la décision d'ouverture de ces deux procédures ne se limite pas à la dette objet du différend uniquement; mais elle s'étend pour concerner l'ensemble des fonds du débiteur actuels et avenirs, ceux relatifs à son commerce ou autres, dans la mesure où le sujet du conflit la dépasse à tous les fonds du débiteur en général (Safae, 1992, p :39-40, Al-Aekali, 2008, p :69).

Si à l'origine la faillite englobe la saisie de tous les fonds du débiteur failli, le législateur saoudien en a excepté les avoirs de l'investisseur étranger en dehors du territoire de l'Etat, il s'agit alors d'encourager les investisseurs à investir au Royaume en les rassurant de ne pas porter préjudice à leurs fonds existants en dehors du Royaume en cas de risque de faillite. Ainsi, le législateur a adopté la théorie de la répartition de l'information financière sur les actifs de l'investisseur, dans le sens où il est possible de séparer les fonds et les actifs de l'investisseur situés dans le royaume et ceux en dehors. 
Si la faillite a pour origine que la faillite englobe tous les fonds du débiteur en faillite, mais que le législateur saoudien a exclu les avoirs de l'investisseur étranger en dehors du territoire de l'État, il serait donc sage d'encourager les investisseurs à investir à l'intérieur du Royaume en les rassurant de ne pas porter préjudice à leurs avoirs en dehors du Royaume S'ils sont en danger de faillite. Le législateur a ainsi adopté la théorie de la répartition de l'information financière sur les actifs de l'investisseur, dans laquelle il est possible de séparer les fonds et les actifs situés dans le royaume et ceux situés en dehors du royaume.

A cet égard, la question se pose des avoirs existants en dehors du royaume dès lors que leur source est principalement les fonds investis dans le royaume? Pour notre part, nous considérons que le législateur saoudien a excepté tous les fonds de l'investisseur en dehors du royaume sans égard à leur source. Nous espérons qu'il maintient l'établissement de l'équilibre entre les intérêts des débiteurs et ceux des créanciers, sans pour autant limiter cette exception aux avoirs qui ne se rapportent pas aux avoirs investis dans le royaume, mais si ces fonds sont étroitement liés aux avoirs à l'intérieur du royaume ; notamment s'ils en sont issus ou en font partie, dans ce cas l'origine c'est qu'ils doivent faire partie de la saisie de l'investisseur en protection aux intérêts des créanciers, et de peur que ce dernier les détourne en dehors du royaume comme précaution contre un risque potentiel de faillite.

Au niveau du paragraphe (27) du premier article, e législateur saoudien a limité les dettes de la saisie du débiteur en: « les dettes fixes révolues du débiteur à la date d'ouverture de l'une des procédures de liquidation prévues par règlement, ou issues de contrats ou engagements qui précèdent cette date, $\mathrm{y}$ compris les dettes du débiteur issues de l'exercice d'activités commerciales, professionnelles ou à but lucratif, ou autres dettes ». Pour dire autrement, ceci inclut toutes les dettes du failli quelles qu'en soit leur nature.

Il importe de mentionner que l'erga omnes du jugement d'ouverture de l'une des procédures de faillite, constitut un moyen justificatif de la fin visée par le règlement de faillite, en général, à savoir la procédure de liquidation collective des fonds du débiteur et leur répartition entre créanciers lorsqu'on ne peut plus sauver le débiteur en difficulté, cette fin ne peut alors être réalisée que si ce jugement est absolument opposable comme nous venons d'expliquer (Al-Aekali, 2008, p:70).

\section{- L'aspect créateur et déclaratif de la décision du tribunal}

La validité du jugement d'ouverture de l'une des procédures de faillite nécessite le rendement de la décision du tribunal compétent, car l'arrêt de paiement uniquement ne suffit pas pour commencer les procédures de faillites sans jugement prononcé à cet effet. Sur cette base, l'ouverture de l'une de ces procédures est un jugement créateur, parce qu'il crée un nouveau statut juridique qui n'existait pas avant son rendement; par exemple, l'ouverture de la procédure de liquidation induit la restriction de la gestion du débiteur à ses biens, la création d'un groupe de créanciers, la déchéance des dettes, l'inefficacité de ses paroles et actes et bien d'autres nouveaux statuts juridiques qui n'apparaissent qu'à la date du jugement.

A cet effet, le débiteur qui cesse d'honorer ses dettes n'acquit pas la qualité de débiteur failli ou en difficulté ou bien qui encoure le risque de faillite que par une décision judiciaire à ce propos, c'est-à-dire que les effets de l'ouverture de l'une des procédures de faillite résultent par force de loi. Alors si ce jugement, d'une part, créateur de l'état de faillite ou de difficulté, d'autre part, il est révélateur de l'état de cessation de paiement ou de risque que le débiteur cesse d'honorer ses dettes, qui précédent le jugement d'ouverture des procédures de faillite.

\section{- La déclaration de l'ouverture de la procédure de faillite}

Etant donné que la décision d'ouverture de la procédure de faillite est un jugement opposable à tous, il est indispensable qu'il soit l'objet de moyens de publicité et de déclaration; afin de tenir au courant toute personne intéressée à ce propos, surtout les débiteurs auxquels la loi impose la présentation de leurs dettes et la souscription aux procédures de saisie. En réalité, la méthode de déclarer la décision d'ouvrir l'une des procédures de faillite varie en fonction du type de procédure à lancer.

S'agissant du cas de la demande d'ouverture de la procédure de règlement préventif, le législateur saoudien exige au débiteur de déclarer le redressement financier pendant 7 jours à compter à partir de la date du rendement de la décision judiciaire de son ouverture. Cependant, qu'en cas de la demande de la procédure de redressement financier ou bien de liquidation normale, dans ce sens, le syndic de faillite désigné par le tribunal se charge de cette déclaration dans un délai de 7 jours à partir de la date de sa désignation en ouverture à la procédure. De même, le législateur saoudien impose au comité de faillite la déclaration du jugement judiciaire décidant l'ouverture de la procédure de liquidation administrative pendant 5 jours comptés à partir de la date de son ouverture.

De ce qui précède nous déduisons que le législateur a imposé la publication de la décision d'ouverture d'une procédure quelconque de faillite tout en déterminant une personne précise au niveau de chaque procédure qui s'en charge, pourtant il n'a pas défini la manière ni le moyen de cette déclaration. A ce propos, il a mentionné sa détermination dans le cadre du règlement d'application. Pour notre part, nous pensons que la déclaration des procédures de faillite doit s'effectuer selon des outils particuliers qui diffèrent des outils de publication des jugements judiciaires normaux, de façon à ce que ces outils soient compatibles avec la nature opposable du jugement d'ouverture de l'une desdites procédures, l'objectif étant donc de tenir au courant tous les créanciers 
ayant endetté le débiteur failli, afin qu'ils puissent consulter le débiteur ou le syndic de faillite ainsi que pour prendre les mesures nécessaires à la protection de leurs droits.

D'ailleurs c'est ce qui a été prévu par le législateur saoudien au niveau du règlement d'application du règlement de faillite, dans la mesure où l'article (6/2) stipule que: «La déclaration se fait par le débiteur, le liquideur judiciaire, ou par le comité de faillite en vertu des dispositions et du règlement d'application sur le site électronique dudit comité ». Cependant que le quatrième paragraphe du même article prévoit que: « Le tribunal devrait ordonner le débiteur, ou le liquideur judiciaire ou même le comité de faillite de déclarer tout jugement ou décision ou procédure».

\section{- Le principe de l'unité de la procédure de faillite}

L'opposabilité absolue du jugement d'ouverture de la procédure de faillite empêche la possibilité d'effectuer une procédure de faillite plusieurs fois simultanément, ainsi que l'établissement de diverses procédures au même temps. Ce qui est connu dans la doctrine par «le principe de l'unité » ou «faillite sur faillite ne vaut». De ce principe découle ce qui suit:

1. Le jugement d'ouverture de la procédure de faillite est rendu par le tribunal de la circonscription où se situe son centre d'activité, et si le débiteur a plusieurs activités principales, on demande l'ouverture de l'une des procédures de faillite à l'un des tribunaux où se situe l'un de ses centres principaux. Il en découle l'abstention des autres tribunaux de statuer des procès qui leur sont présentées, même s'ils soient compétents d'examiner l'affaire.

2. Dans le cas où le débiteur exerce ses travaux et activités de nouveau pendant la saisie puis il cesse d'honorer ses nouvelles dettes une autre fois avant la clôture de la première saisie, les créanciers ne peuvent pas -dans ce cas- demander l'ouverture de la procédure de faillite dudit débiteur une seconde fois; cependant les nouveaux créanciers seront, en conséquence, concernés eux aussi par la saisie en cours. En effet, l'article (77) du règlement d'application énonce que : « Les dettes du débiteur nécessaires à la continuité de son activité et qui se produisent après l'ouverture de la procédure de faillite font partie des charges durant la procédure. Mais, si la saisie finit par un règlement préventif, ou par la dissolution du groupe de créanciers et la liquidation des fonds du débiteur, les créanciers ayant endetté le débiteur ultérieurement, sont autorisés à demander la déclaration de la faillite du débiteur une autre fois (Al-Boustani, 2007, p :121)

\section{Section 2: Le contenu du jugement de la procédure de faillite et les voies de recours}

Si le lancement de l'une des procédures de faillite est tributaire du rendement d'une décision judiciaire à ce propos, il est alors nécessaire que cette décision comporte les informations nécessaires pour prouver la situation ciblée par la procédure concernée ainsi que d'autres informations que le règlement exige; notamment les conditions qui établissent l'état de la procédure, principalement remplir la qualité de débiteur, l'établissement de la situation de cessation d'honoraire, ou la crainte de tomber en difficulté De plus, le règlement a prévu un ensemble de procédures de faillite représentées comme suit:

- La désignation d'un liquidateur judiciaire chargé de l'administration de la saisie et de la gestion des fonds du failli. A cet égard, le tribunal peut désigner un ou plusieurs juges pour l'exécution et la supervision de la procédure du redressement financier et de la liquidation. De même, l'article (53) dudit règlement stipule que: "Le tribunal désigne un ou plusieurs juges -selon sa propre estimation- pour superviser l'exécution de la procédure du redressement financier tout en déterminant ses missions ».

Le liquidateur judiciaire est un représentant du débiteur en vertu du règlement, en ce qui concerne les actions qu'il mène au nom de ce débiteur, comme il est un auxiliaire du tribunal en ce qui relève de son exercice à ses missions et attributions relatives à la procédure; c'est qu'il est interdit de le maltraiter, lui mentir, le désinformer, ou d'interrompre ses missions directement ou indirectement. Aussi, selon le $13^{\text {ème }}$ chapitre du règlement de faillite, ce liquidateur peut demander au tribunal de sanctionner le contrevenant.

- Suite au jugement d'ouverture de la procédure de liquidation et une fois le liquidateur est désigné, le débiteur ne peut plus gérer son activité, de même toute action du débiteur qui porte sur l'un des avoirs saisis est considérée nulle. En effet, le liquidateur remplace le débiteur en termes de gestion de son activité et de se conformer à ses obligations pendant la durée de la procédure.

- Le liquidateur judiciaire est tenu prendre les mesures nécessaires pour protéger les droits des créanciers ainsi que de porter intérêt à l'établissement des devoirs du débiteur et de recevoir toutes les dettes qu'il doit, tout en prenant les mesures qu'il estime convenable pour vendre les biens saisis à un meilleur prix.

- L'ouverture de la procédure de liquidation implique le début de liquidation des biens à saisir, à ce niveau le liquidateur judiciaire se charge de leur liquidation, excepte ce que juge le tribunal ayant un caractère naturel pour assurer au débiteur le suffisant pour subvenir à ses besoins et à ceux de sa famille.

En raison de la spécificité qui marque les jugements et les décisions relatives aux procédures de faillite ainsi que de leur opposabilité à tous s'ils n'en sont pas partis, le législateur saoudien a prévu des règles spéciales concernant l'opposition aux dispositions et aux jugements relatifs à la faillite et ceci dans les articles (215-218). Cependant, la manière de s'y opposer diffère en fonction de la partie qui l'émette: 
1. Les procédures et les décisions émises par le débiteur, le créancier, le liquidateur ou la partie compétente et le comité de faillite, peuvent faire l'objet d'oppositions en vertu de l'article (215) du règlement de faillite, et ceci devant le tribunal compétent à statuer des affaires de faillite. Cette opposition doit être présentée pendant (14) jours à compter de lu rendement de la décision ou de lancement de la procédure, exception faite à ce stade de l'autorisation des liquidateur judiciaires et des experts. Dans ce cadre, le jugement définitivement rendu par le tribunal et non susceptible de recours, sauf qu'il fasse partie des dispositions pouvant être contestées devant la cour d'appel.

2. La remise en cause des jugements et des décisions rendus par le tribunal est possible en vertu de l'article $(217 / 1)$ du règlement de faillite qui permet à toute partie ayant intérêt de s'opposer devant la cour d'appel au jugement du tribunal compétent de statuer des affaires de faillite, et ceci dans les cas définis par l'article précité; notamment le refus d'ouverture de la procédure de règlement préventif, ou du redressement financier, etc. L'opposant doit présenter son opposition durant les (14) jours suivant le rendement du jugement ou de la décision. A cet égard, la cour d'appel peut soit confirmer le jugement ou la décision sujet (te) d'opposition soit l'annuler par un jugement définition non susceptible de recours.

\section{Conclusion}

A travers cette étude nous avons abouti à certains résultats et recommandations que nous formulons comme suit:

\section{Premièrement: les résultats}

- Le législateur saoudien n'a pas exigé dans son nouveau règlement de faillite, le rendement d'un jugement de déclaration de la faillite du débiteur en cas de faillite effective ou de difficulté du projet économique; en conséquence il a, ainsi, dérogé aux règles générales de faillite qui prévoient que: «Nulle faillite sans jugement judiciaire qui la décide ».

- Le champ d'application du règlement de faillite ne se limite plus aux commerçants seuls; mais il concerne aussi toutes les personnes, les entités et les institutions à but lucratif; ce qui signifie la possibilité de faillite des personnes non commerçantes, et qui continuent d'exercer des activités professionnelles, industrielles, civiles ou autres. En conséquence, en ce qui relève des affaires de faillite, ceux-ci sont soumis au tribunal de commerce.

- Le législateur saoudien a divisé les jugements judiciaires de faillite en des jugements qui varient selon les procédures de faillite, par exemple, si le débiteur est en situation de difficulté d'honorer ses dettes à terme, il peut demander au tribunal l'un des jugements de faillite, à commencer par celui de la procédure du règlement préventif jusqu'à celle de liquidation des fonds du débiteur failli.

- Le droit de demander l'ouverture de l'une des procédures de faillite varie d'une procédure à une autre; c'est qu'il peut se limiter dans certaines au débiteur en difficulté ou failli, comme il peut concerner dans d'autres le débiteur et le créancier ainsi que de la partie compétente. Aussi, ce droit peut relever du tribunal luimême qui peut décider de l'ouvrir de sa propre initiative, particulièrement suite à l'échec du règlement préventif ou du redressement financier pour les raisons déterminées par le législateur. En contrepartie, ce règlement est exempt de tout droit de la procurature générale à demander le lancement de certaines procédures de faillite.

\section{Deuxièmement: les recommandations}

- En raison de la spécificité marquant le jugement de faillite, et qui le distingue de tout autre jugement judiciaire, particulièrement dans le cadre de la multitude des procédures de faillite, de son champ d'application qui englobe aussi bien les commerçants que d'autres personnes et entités à but lucratif ; nous souhaitions que le législateur saoudien créerait une juridiction dédiée aux affaires de faillite, tout en la dotant des compétences judiciaires spécialisées, dont la mission est seulement d'examiner les affaires de faillite et les affaires qui en découlent.

- $\quad$ Nous proposons au législateur saoudien d'amender la clause (c) du second paragraphe de l'article (93) relative à la vérification de l'authenticité des dettes, de sorte à ne pas la limiter à des outils bien déterminés; pour qu'elle devient comme suite: « que la dette qu'on demande de rembourser soit dû en vertu d'un titre exécutif, ou bien d'un écrit normal, ou tout autre moyen si la dette est commerciale ... ».

- Nous espérions également le fait que le législateur saoudien ait stipulé explicitement la demande d'ouverture de l'une des procédures de faillite auprès du tribunal du lieu d'exercice de l'activité à but lucratif du débiteur et non pas de son lieu de résidence habituel. 


\section{Bibliographie:}

\section{Bibliographie en langue arabe:}

[1] Al-Aekali. Aziz, Le médiateur d'explication du droit commercial, Les jugements de faillite et sa réconciliation préventive, troisième partie, Maison d'édition Dar At-Thaqafa/ Oman, (2008).

[2] Al-Baroudi. Ali, Le sommaire du droit commercial: la faillite et les papiers commerciaux, Le bureau égyptien moderne, Alexandrie, (1968).

[3] Al-Boustani. Said, Les dispositions de la faillite et la réconciliation préventive dans les législations arabes, Publications Dar Al-Halabi, Liban, (2007).

[4] Ahmad. Ibrahim, L'erga Omnes des jugements, Dar Al-Fikr Al-Jamii, Alexandrie.

[5] Karman. Mohamed Sayid, Les papiers commerciaux, la faillite et le règlement préventif, Librairie Chqiri, deuxième édition, Riad, 1429 de l'hégire.

[6] Safae. Aili, Les jugements de faillite et de conciliation preventive, Dar Al-Manchourat Al-Hoqouqia, (1992).

[7] Taha. Moustafa Kamal, Le droit commercial: les papiers commerciaux et la faillite, Dar Aljamiaa/ Bayrouth, s.d.

\section{Bibliographie en d'autres langues:}

[1] Boughanmi. Afef, et les autres, Analyse économique du droit de la faillite: les dix fonctions des procédures collectives, Dans Revue d'économie financière (2018).

[2] George. Lloyd D., From Orphan to Maturity: The Development of the Bankruptcy System During L. Ralph Mecham's Tenure as Director of the Administrative Office of the United States Courts L. Ralph Mecham \& Federal Courts Administration: A Decade of Innovation and Progress, American University Law Review 44, no. 5 (June 1995): 1491-1501.

[3] HOUIN. C., Permanence de l'entreprise à travers la faillite, Liber amicorum, baron Louis Frédéricq, (1965). 